\title{
TRANSFER IN GENERALIZED SHEAF COHOMOLOGY
}

\author{
ROBERT PIACENZA
}

\begin{abstract}
The aim of this note is to define the transfer in generalized sheaf cohomology and state its most important properties. Under appropriate conditions the transfer defined here agrees with the transfer defined using different methods by Roush, Kahn, and Priddy.
\end{abstract}

Transfer for finite coverings. In order to formulate our main theorem (1.1) we need some basic definitions involving

1.0 Generalized sheaf cohomology and finite coverings. When speaking of sheaves of spectra we shall adopt the language of Brown as formulated in [4 and 5]. Let $\mathcal{E}$ be a sheaf of spectra over $B$. Unless otherwise stated we shall assume that $\mathcal{E}$ is in $\mathrm{Sta} \mathrm{Ho}^{+}(B)$ or that $B$ has finite cohomological dimension. Let $f: X \rightarrow B$ be a continuous map and $\phi$ a family of supports on $B$. We let

(i) $f^{*}: H_{\phi}^{*}(B ; \mathcal{E}) \rightarrow H_{f^{-1} \phi}^{*}\left(X ; f^{\#} \mathcal{E}\right)$ be the natural homomorphism in cohomology induced by the natural $f$-cohomomorphism.

(ii) $f^{\#} \mathcal{E} \cdot \rightarrow \mathcal{E}\left(f f^{\#}\right.$ denotes pullback). The cohomology group on the right in (i) will be written $H_{\phi}^{*}(X ; \mathcal{E})$ with $f$ understood. The map $f$ is called a finite covering map if it is a locally trivial bundle projection with discrete finite fiber. If $U \subseteq B$ and $f^{-1}(U)=W \subseteq X$, then we call $f:(X, W) \rightarrow(B, U)$ a finite covering map of pairs.

1.1 Existence TheORem. For each finite covering map $f$ there exists an abelian group homomorphism $f^{\prime}: H_{\phi}^{*}(X ; \mathcal{E}) \rightarrow H_{\phi}^{*}(B ; \mathcal{E})$ called transfer in $\mathcal{E}$ cohomology with the following properties:

1.2 Transfer is functorial over pullbacks of coverings.

1.3 Transfer for an identity covering is the identity.

1.4 If a covering splits as a disjoint union of coverings, then transfer over the total covering is the sum of the transfers over the component covering.

1.5 Transfer for a composition of coverings is the composition of the transfers.

1.6 Transfer commutes with homomorphisms induced by a morphism of coefficient sheaves.

1.7 Transfer induces a morphism on the $E_{2}$ terms of the generalized Atiyah-Hirzebruch spectral sequence [5, Theorem $8, p .451]$ and is compatible with the differentials of that sequence. (We do not assume here that $\mathcal{E} \in \mathrm{Sta} \mathrm{Ho}^{+}(B)$.)

Received by the editors April 23, 1982. Presented to the winter meeting of the AMS in Denver in January 1983.

1980 . Mathematics Subject Classification. Primary 55N30.

Key words and phrases. Transfer, sheaf, spectra, Postnikov decomposition, finite covering map, hypercovering, generalized Čech cohomology. 
1.8 Transfer is compatible with cup products induced by pairings of spectra (see [5, Appendix A]).

1.9 If $U$ is open or if $U$ is closed and $B$ is paracompact then there is a transfer $f !$ : $H^{*}(X, W ; \mathcal{E}) \rightarrow H^{*}(B, U ; \mathcal{E})$ that agrees with our previous transfer if $U$ is empty. Furthermore, $f^{\prime}$ is compatible with the coboundary operator of a pair.

1.10 Transfer is compatible with the spectral sequence of an open covering (or hypercovering) of $B$ as defined in [5, Remark 2, p. 450]. In particular, transfer is compatible with suitable Mayer-Vietoris sequences (see [5, Proposition 11, p. 449]).

The proof of 1.1 is given below. To prove 1.1 we need

1.11 Proposition. Let $f_{\#}: \operatorname{Sp}(X) \rightarrow \operatorname{Sp}(B)$ be the direct image functor and $f^{\#}$ : $\mathrm{Sp}(B) \rightarrow \mathrm{Sp}(X)$ the pullback functor where $f$ is a finite covering. Then $f_{\#}$ preserves weak equivalences, local fibrations, fibration sequences, global fibrations and smash products. $f^{\#}$ preserves weak equivalences, local fibrations, fibration sequences and smash products.

Proof. The proof is easy and left to the reader.

To define the transfer, consider the sheaf $\mathcal{E}^{1}=f_{\#} f^{\#} \mathcal{E}$. There is a subsheaf $i$ : $\varepsilon^{2} \rightarrow \mathcal{E}^{1}$ with stalk $\varepsilon_{b}^{2}=\bigvee \mathcal{E}_{b}$ where on the right we have a coproduct of card $f^{-1}(b)$ copies of $\varepsilon_{b}$. Now by $[1,3.14$, p. 157] $i$ is a weak equivalence in $\operatorname{Sp}(B)$. Let $s$ : $\varepsilon^{2} \rightarrow \mathcal{E}$ be the natural map; i.e., on each stalk $s$ is a coproduct of identities. Thus we have a diagram

$$
\mathcal{E}^{1} \stackrel{i}{\leftarrow} \mathcal{E}^{2} \stackrel{s}{\rightarrow} \mathcal{E} .
$$

By 1.11 there is a natural isomorphism $H_{\phi}^{*}(X ; \mathcal{E}) \simeq H_{\phi}^{*}\left(B ; \mathcal{E}^{1}\right)$. Thus we have the following diagram:

$$
H_{\phi}^{*}(X ; \mathcal{E}) \simeq H_{\phi}^{*}\left(B ; \mathcal{E}^{1}\right) \simeq H_{\phi}^{*}\left(B ; \mathcal{E}^{2}\right) \stackrel{s_{*}}{\rightarrow} H_{\phi}^{*}(B ; \mathcal{E}) .
$$

We now use $s_{*}$ to define $f !$ in the obvious way.

We now turn to the proof of 1.1. One easily checks $1.2-1.6$ using 1.11 and suitable properties of sheaf cohomology. 1.7 follows from the fact that $f_{\#} f^{\#}$ preserves the Postnikov decomposition of $\mathcal{E}$ as defined in [5, p. 446]; i.e., the $N$ th Postnikov approximation of $\mathcal{E}$ goes under $f_{\#} f^{\#}$ to the $N$ th Postnikov approximation of $\mathcal{E}^{1} .1 .8$ follows directly from 1.11 using the definition of cup product given in [5, Appendix A, p. 451]. 1.9 follows from 1.11 in view of the definition of relative cohomology in $[5, \S 11$, p. 449]. 1.10 is easy to check using 1.11 again.

2.0 Generalized Čech cohomology and finite coverings. In this section we remove the restrictions placed on $\mathcal{E}$ in $\S 1$. Let $\mathcal{E}$ be in $\operatorname{Sp}(B)$. We define the Čech cohomology of $B$ with coefficients $\mathcal{E}$ denoted $\check{H}^{*}(B ; \mathcal{E})$ by

$$
\check{H}^{q}(B ; \mathcal{E})=\lim _{K \in \overrightarrow{H R}(B)} \Pi\left(K, \underline{\mathcal{E}}_{(q)}\right)
$$

where $H R(B)$ is the category of hypercoverings of $B$ as defined in [5, Theorem 2, p. 427 or $2, \S 8], \Pi\left(K, \mathcal{E}_{(q)}\right)$ is the set of simplicial homotopy classes of maps in $\delta(B)$; 
i.e., the category of sheaves of simplicial sets over $B$ and $\mathcal{E}_{(q)}$ is defined as in [5,

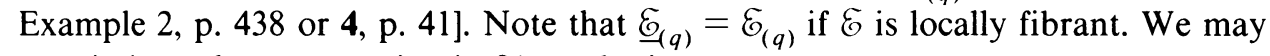
now imitate the construction in $\$ 1$ to obtain

2.1 Proposition. For each finite covering map $f: X \rightarrow B$ there exists an abelian group homomorphism $f^{!}: \check{H}^{*}(X ; \mathcal{E}) \rightarrow \check{H}^{*}(B ; \mathcal{E})$ called the transfer in generalized Cech cohomology that satisfies $1.2-1.6$ of 1.1 .

2.2 REMARK. (i) If $\mathcal{E}$ is in $\mathrm{Sta} \mathrm{Ho}^{+}(B)$ or $B$ has finite cohomological dimension, then there is a natural isomorphism $\check{H}^{*}(B ; \mathcal{E}) \simeq H^{*}(B ; \mathcal{E})$; i.e., Verdier's hypercovering theorem [4, Corollary 1.8, p. 53]. It follows that transfer in Čech cohomology specializes to the transfer defined in $\S 1$ under the given assumption on $\mathcal{E}$ or $B$. (ii) If $U \subseteq B$ is open we define the relative Čech cohomology by

$$
\check{H}^{q}(B, U ; \mathcal{E})=\lim _{K \in \overrightarrow{H R}(B)} \Pi\left(K, K \mid U ; \underline{\mathcal{E}}_{(q)}, *\right)
$$

where $K \mid U$ is $K$ restricted to $U, *$ is the natural base point cross section and $\Pi$ refers to homotopy classes of maps of pairs. One may easily extend the definition of transfer in Čech cohomology given above to transfer in relative Čech cohomology.

\subsection{Comparisons with other definitions of transfer.}

3.1 The transfer of Roush-Kahn-Priddy. In [6 and 7] a theory of transfer in generalized cohomology is developed for the category of $\mathrm{CW}$-complexes. We extend their definition of transfer to all spaces via the singular complex functor $S$.

Let $\mathcal{E}$ be in $\mathrm{Sp}(\mathrm{pt}$.). If $B$ is completely paracompact and locally contractible then there is a natural isomorphism

$$
\begin{aligned}
\check{H}^{q}(B, U ; \mathcal{E}) & \simeq H_{\text {sing }}^{q}(B, U ; \mathcal{E}) \\
& =H^{q}(S(B), S(U) ; \mathcal{E})=\Pi\left(S(B), S(U) ; \underline{\mathcal{E}}_{(q)}, *\right)
\end{aligned}
$$

where $U$ open $\subseteq B, \mathcal{E}$ is the constant sheaf over $B$ and $H_{\text {sing }}^{*}$ is the generalized singular cohomology functor. This may be proved using [2, Theorem 12.1, p. 129] (see also [4, Remark 1.10, p. 54]). We may use the above isomorphism to define the long exact sequence of a pair $(B, U)$ and appropriate Mayer-Vietoris sequences in generalized Čech cohomology. One easily checks that transfer in Čech cohomology as defined in 2.2(ii) is compatible with the coboundary operator of such sequences.

The following result is a direct corollary to the uniqueness theorem of Lewis [8, Theorem 1.2, p. 91].

3.2 Proposition. If $B$ is completely paracompact and locally contractible (this is satisfied if $B$ is a $C$. W. complex) the transfer in generalized Cech cohomology with constant coefficients agrees with the transfer of Roush-Kahn-Priddy if the transfers are viewed as maps into $\lim _{n} H^{*}\left(S(B)_{n} ; \mathcal{E}\right)$ where $S(B)_{n}$ is the $n$-skeleton of $S(B)$.

3.3 The transfer in ordinary sheaf cohomology. Let $\mathbb{Q}$ be a sheaf of abelian groups over $B$ and $f: X \rightarrow B$ a finite covering map. Let $Q^{1}=f_{\#} f^{\#} Q$. We define a homomorphism of sheaves $\Sigma: \mathbb{Q}^{1} \rightarrow \mathbb{Q}$ as follows: On stalks $\Sigma$ is given by summation $\Sigma_{b}: \mathbb{Q}_{b}^{1}=\bigoplus \mathbb{Q}_{b} \rightarrow \mathbb{Q}_{b}$ where $\oplus \mathbb{Q}_{b}$ is a sum of card $f^{-1}(b)$ copies of $\mathbb{Q}_{b}$. 
3.4 Proposition. $\Sigma$ induces a map $f^{!}: H_{\phi}^{*}(X ; \mathbb{Q}) \rightarrow H_{\phi}^{*}(B ; \mathbb{Q})$ called transfer in ordinary sheaf cohomology. Furthermore this transfer satisfies an appropriate version of 1.1 .

Proof. The proof is left to the reader.

3.5 REMARK. (i) We note that $f^{\prime} f^{*}(u)=r u$ where $u \in H_{\phi}^{*}(B$; $\mathbb{Q}$ ) and $r$ is the index of the covering $f$. (Assuming here that for all $b \in B$, card $f^{-1}(b)=r$.) (ii) Compare transfer in ordinary sheaf cohomology to the transfer defined in [3, Chapter II, 19, p. 83]. (iii) If $\mathcal{E}$ is a sheaf of abelian group spectra in $\operatorname{Sp}(B)$, then we may use the above map $\Sigma$ to define transfer in the hyper-cohomology groups of $\varepsilon$. See [5, Example 2, p. 448] for the definition of these groups.

Now let $\mathcal{E}$ be an abelian group spectrum in $\operatorname{Sp}(B)$. For example, $\mathcal{E}$ could be $K(Q, 0)$; i.e., the Eilenberg-Mac Lane spectrum of the abelian sheaf $\mathcal{Q}$ as defined in [5, Lemma, p. 450]. In the notation of $\S 1$ we observe that $s=\Sigma i$. The following result is thus immediate.

3.6 Proposition. Transfer in ordinary sheaf cohomology agrees with the transfer defined in $\S 1$.

To formulate our next result we need the following definition. Let $P$ be a set of primes. A sheaf of spectra over $B$ is called $P$-local if $\Pi_{*}(\mathcal{E})$; i.e., the sheaf of stable homotopy groups of $\mathscr{E}[5$, p. 441] is a sheaf of $P$-local abelian groups. We note that if $\mathcal{E}$ is $P$-local then so is its $N$ th Postnikov approximation for all $N$.

3.7 Proposition. Let $\mathcal{E}$ be in $\mathrm{Sta}^{+} \mathrm{Ho}^{+}(B)$ or $B$ have finite cohomological dimension. Let $\varepsilon$ be $P$ local and $f: X \rightarrow B$ a finite covering map of index $r$. If each prime that divides $r$ is not in $P$, then the endomorphism $f^{!} f^{*}: H^{*}(B ; \mathcal{E}) \rightarrow H^{*}(B ; \mathcal{E})$ is an isomorphism. In particular, $f^{*}$ is a monomorphism and $f^{!}$is an epimorphism.

PROOF. The proof is an easy application of 1.7 and 3.5(i).

\section{REFERENCES}

1. Frank Adams, Stable homotopy and generalized homology, Univ. of Chicago Press, Chicago, Ill., 1974.

2. M. Artin and B. Mazur, Etale homotopy, Lecture Notes in Math., vol. 100, Springer-Verlag, Berlin and New York, 1969.

3. Glen Bredon, Sheaf theory, McGraw-Hill, New York, 1967.

4. Kenneth Brown, Abstract homotopy theory and generalized sheaf cohomologv, Thesis, MIT, 1971.

5. Abstract homotopy theory and generalized sheaf cohomologv, Trans. Amer. Math. Soc. 186 (1973), 419-458.

6. Daniel Kahn and Stewart Priddy, Applications of the transfer to stable homotopy, Bull. Amer. Math. Soc. 78 (1972), 981-987.

7. F. W. Roush, Transfer in generalized cohomology theories, Thesis, Princeton Univ., 1971.

8. L. G. Lewis, Jr., The uniqueness of bundle transfers, Math. Proc. Cambridge Philos. Soc. 93 (1983), 87-111.

Department of Mathematics, University of Alaska, Fairbanks, Alaska 99701 